\title{
Development of a Precise Magnetic Field Measurement System for Fast-Changing Magnetic Fields
}

\author{
Peter Wanderer, John Escallier, George Ganetis, Animesh Jain, Wing Louie, Andrew Marone, Richard Thomas
}

\begin{abstract}
Several recent applications for fast ramped magnets have been found that require precise measurement of the timedependent fields. In one instance, accelerator dipoles will be ramped at $1 \mathrm{~T} / \mathrm{sec}$, with measurements needed to the typical level of accuracy for accelerators, $\triangle B / B$ better than $0.01 \%$. To meet this need, we have begun development of a system containing 16 stationary pickup windings that will be sampled at a high rate. It is hoped that harmonics through the decapole can be measured with this system. Precise measurement of the time-dependent harmonics requires that both the pickup windings and the voltmeters be nearly identical. To minimize costs, printed circuit boards are being used for the pickup windings and a combination of amplifiers and ADC's for voltmeters. In addition, new software must be developed for the analysis. The paper will present a status report on this work.
\end{abstract}

Index Terms-fast magnetic field measurements, non-rotating pickup coil, printed circuit boards

\section{INTRODUCTION}

$\mathrm{M}$ AGNETIC field measurements of nearly all accelerator magnets are made with systems designed for constant or slowly-varying fields. Measurements of multipoles are commonly made with rotating coils that have a period of several seconds [1]. Recently, we have encountered a need for measurements of fast-ramped fields in magnets for three different projects. A significant upgrade has been approved for the accelerator facility at Gesellchaft fuir Schwerionenforschung (GSI) in Darmstadt, Germany. The upgrade will include two superconducting synchrotrons that will ramp at $d B / d t 1-2 \mathrm{~T} / \mathrm{s}$ [2]. A model fast-ramp dipole has been built and operated at this ramp rate at BNL by a BNLGSI collaboration [3]. BNL is exploring whether the Alternating Gradient Synchrotron (AGS) can be ramped at high rates so that a next-generation neutrino beam can be produced [4]. Also at BNL, a model magnet that will

Manuscript reccived October 20, 2003. This work was supported by GSI and the U.S. Dept. of Energy under Contract No. DE-AC02-98CH10886.

P. Wandercr (cmail: wandercr(abnl.gov), J. Escallicr (cmail: escallic(abnl.gov ). G. Ganctis (cmail: ganctis1@bnl.gov), A. Jain (cmail: iain(obnl.gov), W. Louic (cmail: louic@bnl.gov), A. Maronc (cmail: andym(abnl,gov), and R. Thomas (cmail: thomasl(cobnl,gov) are with the Superconducting Magnet Division, Brookbaven National Laboratory, PO Box 5000, Upton. NY 11973-5000 USA. generate orthogonal $0.4 \mathrm{~T}$ dipole fields using two $\cos -\theta$ windings has been built and ramped successfully at rates of several $\mathrm{Hz}$. Magnetic ficld measurements are needed to test whether the field can track the motion of small mammals, such as a rat [5]. If so, a magnet like this could be used inside a $4 \mathrm{~T}$ NMR solenoid to potentially allow scans of awake animals. (At present, NMR scans can only be made on sedated animals.) For each of these magnets, it is important to measure all the low-order harmonics.

In certain instances, measurements of the time variation of specific harmonics (e.g., the normal sextupole) have been made with specialized systems. For example, a coil sensitive to a single multipole, such as the sextupole, can make measurements at a few $\mathrm{Hz}$. Such devices are of interest for measuring the time-dependent behavior of the sextupole in superconducting accelerator magnets. Limitations on coil rotation speed and signal sampling prevent its use for the systems described above.

A non-rotating coil with multiple probes can be used to make measurements at higher rates. For example, a system of three Hall probes can be set up to measure the sextupole. However, careful intercalibration of each probe is necessary, and nonlinearities limit the accuracy of the device. A system of multiple pulsed NMR probes has been successfully used in DC magnets, but measurements of rapidly varying multipoles have not been demonstrated [6,7]. Furthermore, such a system is significantly more complex as compared to search coils; and can only be used for homogeneous fields, such as dipoles with good field quality.

\section{GENERAL APPROACH}

We are developing a harmonic coil array for measuring fastramped magnets. Printed circuits are relatively inexpensive and reproducible, and will be used for the windings. Sixteen printed circuits will be mounted as tangential coils on a cylinder. We are sampling terms as high as the 16-pole, with the goal of obtaining reliable measurements at least as high as the decapole. The 16 probes will be intercalibrated by rotating the coil in a reference dipole field. However, the coil will be stationary when used for measuring fast-ramped fields.

The voltage will be sampled at rates as high as $50 \mathrm{~Hz}$. The voltage sampling system must also cover a wide range of signal amplitudes and allow for frequent calibration and DC 
offset checks.

In the stationary mode, the total flux seen by a given probe is determined by its angular position, as well as the multipole components of the field. The angular profile constructed with voltage signals from various probes is, in general, not a Fourier series. In order to simplify the analysis, it is essential that the geometric parameters of various probes be as identical as possible, and the probe angular positions be as uniformly spaced as possible (see Sec. V).

\section{PRINTED CIRCUIT PROBES}

We have designed a 10-layer printed circuit board with six turns per layer for a total of 60 turns. The lines on the circuit boards are $0.1 \mathrm{~mm}$ wide and have $0.1 \mathrm{~mm}$ gaps between them. The circuit boards are $0.3 \mathrm{~m}$ long and the average width of the pattern is $11.6 \mathrm{~mm}$. Fig. 1 shows the probe array assembled for the bio-med magnet and a detail of one of the printed circuit cnds. The boards are $1.7 \mathrm{~mm}$ thick and the effective radius of the cylinder on which the probes are mounted is $35.7 \mathrm{~mm}$.

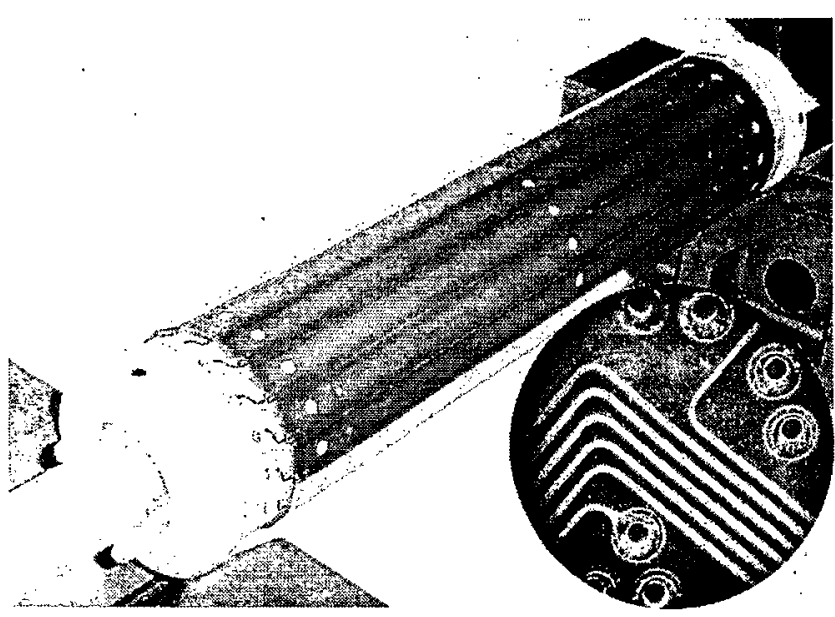

Fig. I. Array of 16 printed circuits mounted on a cylindrical coil form. The inset shows the detail in a corner.

\section{A. Probe Inspection}

Several parameters have been measured for inspection of the printed circuits: probe resistance, probe inductance, and voltage generated in a ramped dipole field. Careful and efficient inspection was necessary because a significant fraction of the probes had turn-to-turn shorts. In principle, probes with turn-to-turn shorts can be identified by measuring the resistance of each one. However, experience showed that the variation of the resistance of the probes themselves was large enough to mask shorted turns. This is primarily because cven small differences in the copper thickness can produce a relatively large percentage change in the resistance. Inductance measurements were found to be a much more reliable indicator of the total probe area. This can be seen in Fig. 2, where there is a clear separation between GSI probes with $\mathrm{L} \sim 1.13 \mathrm{mH}$ and those with $\mathrm{L}<1.10 \mathrm{mH}$. (These probes have not yet been chccked in a dipole field, as described below.)

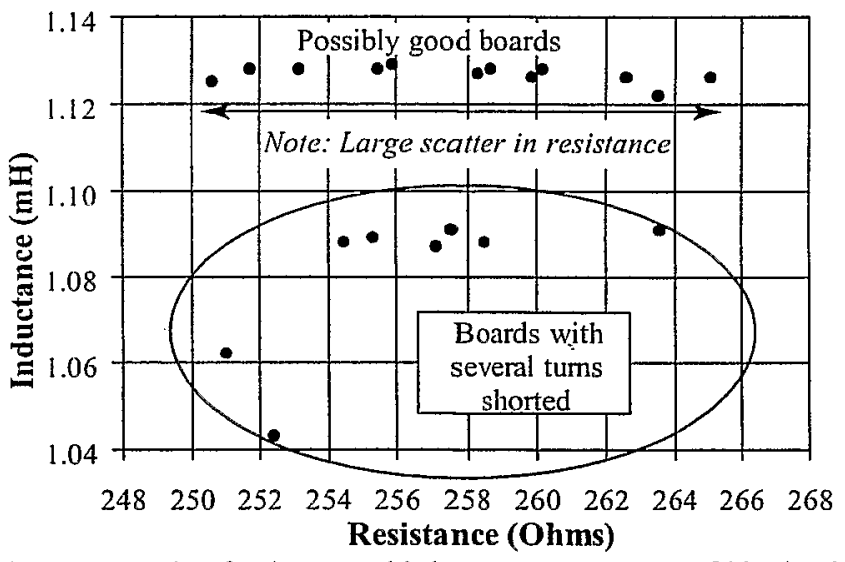

Fig. 2. Scatter plot of resistance and inductance measurements of 20 printed circuits for the GSI coil.

To inspect the probes in a ramped magnetic field, the probes were mounted side-by-side on a flat plate and placed in a large-aperture dipole. The dipole was ramped at $\sim 0.1 \mathrm{~T} / \mathrm{s}$ for several seconds and the signal from each of the windings was recorded. Fig. 3 shows the results for four Biomed probes two good ones, one with a single shorted turn, and one with a shorted layer. Inductance measurements on the same boards were in qualitative agreement with these results, but were not accurate enough to allow a precise matching of probe area. For example, coil- 4 shows a $1.75 \%$ reduction in the probe area from the field measurements, corresponding to a single shorted turn, but shows a $3.2 \%$ reduction in inductance. Similarly, field measurements show a reduction of $8.2 \%$ in the area of coil-3, but the inductance measurements show a drop of $14.6 \%$. Nevertheless, the inductance measurements being much simpler to perform, we have adopted these measurements as the basis for a quick inspection. The boards that appear matched in inductance will then be further tested in a ramping magnetic field for a more precise matching of the total surface area.

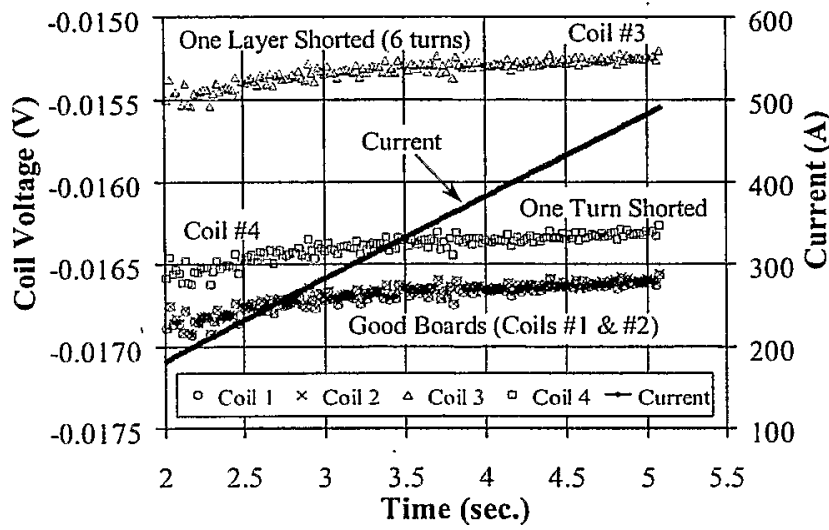

Fig. 3. Plot of the voltage gencrated versus time in four non-rotating Biomed probes in a ramping dipole ficld. The plot also shows the magnet current vcrsus timc.

We have inspected 40 probes so far, and have found 10 of them to be defective. It has been possible to repair only a few 
of them where the shorts were in the outermost layers. The problems found with the probes are similar to those found in an earlier use of printed circuits to measure magnetic fields [8].

\section{B. Probe Calibration}

The probes were calibrated by rotating the probe array in a constant dipole field, recording the output simultaneously and analyzing the data with an FFT. Fig. 4 shows the outputs. The high-frequency variation seen in each signal is due to vibrations of the rotating coil. Since the frequency of the vibration is much larger than that of the coil rotation, it does not affect the lowest (dipole) term of the FFT, which is used for calibration. The coil bearings are being modified to reduce the vibration. It should be noted that for fast measurements. the probe will be used in a stationary mode and the vibration is not a problem.

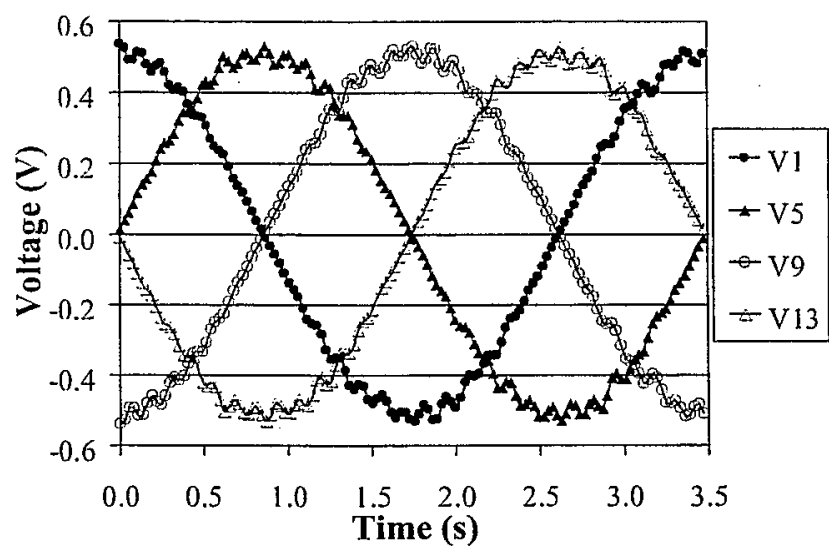

Fig. 4. Plot of the voltages generated by some of the 16 Biomed probes mounted on a support cylinder that rotates with a period of $3.5 \mathrm{~s}$ in a constant dipole ficld of $1.37 \mathrm{~T}$. The signal amplitude corrcsponds to a ramp ratc of $2.46 \mathrm{~T} / \mathrm{s}$ in the stationary mode.

Fig. 5 shows the calibration results for 16 probes. The difference between the maximum and the minimum responses is nearly $0.06 \%$, somewhat larger than desired for a simplified analysis method. To reduce the spread in probe responses, additional probes have been ordered. Since the probes are inexpensive, sorting will be used to obtain the desired uniformity of response, preferably with a standard deviation of $\sim 0.01 \%$. The phases of the dipole signals are used to calibrate the angular positions of the various windings.

\section{DATA ACQUISITION SYSTEM}

To minimize the cost of the data acquisition system, we purchased digital multimeters with the required measurement accuracy but a minimum of extra capability (HP 34401). However, these meters do not allow sufficient control of the time of the trigger for measurements. The variation of the timing leads to inaccurate measurements of fast-changing signals. This can be seen in Fig. 6 , which displays the signals recorded with the same $10 \mathrm{~V}, 4 \mathrm{~Hz} \sin$ wave input to all the meters and the difference between two of them, $\sim 10 \mathrm{mV}$. (For this measurement, the display was turned off to minimize the variation in trigger time.)

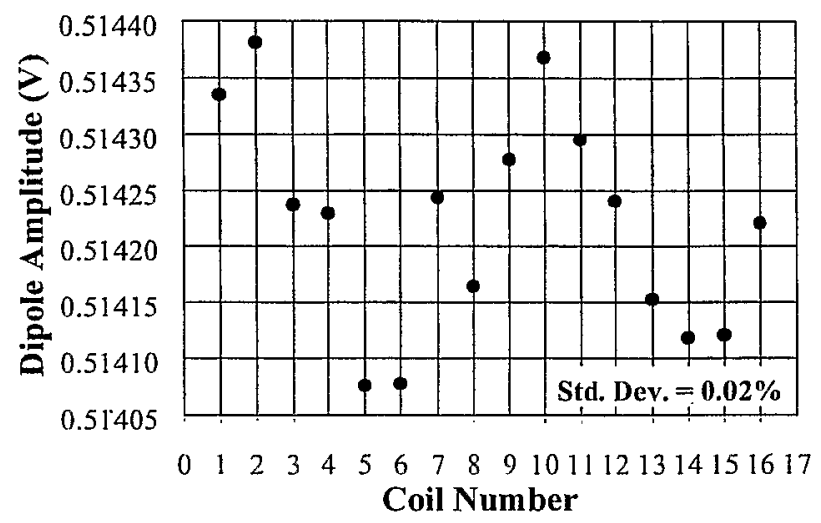

Fig. 5. Dipole amplitudes from the FFT of the 16 rotating probe voltages in a fixed dipole ficld of $1.37 \mathrm{~T}$.

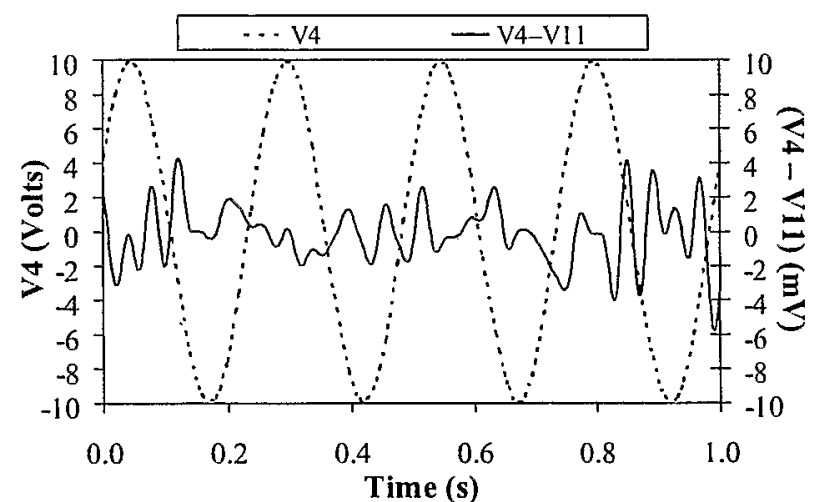

Fig. 6. Voltage input to cach of the multimeters and recorded by onc of them (left ordinatc) and typical voltage difference between two of them (right ordinate) versus time.

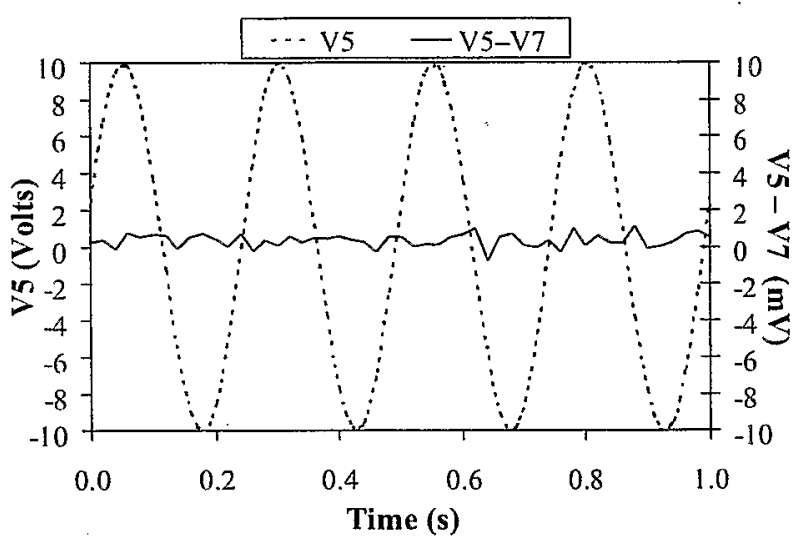

Fig. 7. Voltage input to cach of the ADC's and recorded by one of them (left ordinatc) and typical voltage difference betwecn two of them (right ordinate) versus time.

Our present plan is to use an array of 16-bit ADC's and build programmable gain amplifiers in order to obtain the factor of $\sim 100$ gain needed for some of the measurements. Fig. 7 shows the results of an ADC measurement of the same signal as that shown in Fig. 6. With improved timing, the variation between $\mathrm{ADC}$ channels is $\sim 1 \mathrm{mV}$. 
The programmable gain amplifiers are under construction. For stability, they will be mounted in a temperature-controlled cnvironment. The system will be designed so that a calibration run will precede every data acquisition run. A test of the complete system is planned for later this year.

\section{DATA ANALYSIS}

For the purpose of data analysis, a coordinate system internal to the coil is used. The X-axis is chosen along the centerline of one of the probes, as shown in Fig. 8. Since the rclative angles of all the probes are determined by rotating the coil in a dipole field, the angular positions, $\theta_{i}$, of all the probes arc known in this coordinate system. In practice, it is desirable to align this coordinate system as best as possible with the magnet's principle axes. A perfect alignment, however, is not cssential, and will not be assumed in the data analysis.

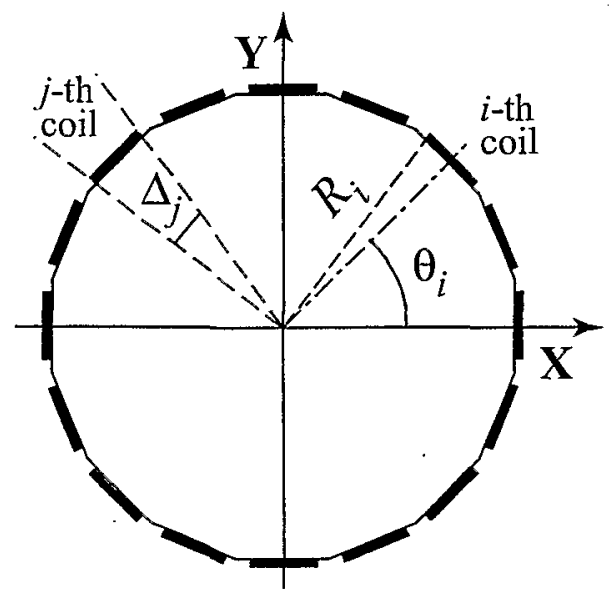

Fig. 8. Coordinate system used for analysis of data in the stationary mode.

The flux linked at time $t$ through the $i$-th tangential coil of length $L_{i}$ and having $N_{i}$ turns is given by

$$
\begin{aligned}
\Phi_{i}(t) & =\sum_{n=1}^{\infty} \frac{2 N_{i} L_{i} R_{r e f}}{n}\left(\frac{R_{i}}{R_{r c f}}\right)^{n} \sin \left(\frac{n \Delta_{i}}{2}\right) \\
& \times\left[B_{n}(t) \sin \left(n \theta_{i}\right)+A_{n}(t) \cos \left(n \theta_{i}\right)\right]
\end{aligned}
$$

where $B_{n}(t)$ and $A_{n}(t)$ are the normal and skew $2 n$-pole terms respectively at a reference radius of $R_{r e f}$ in a harmonic expansion of the 2-D field components. The induced voltage in the $i$-th probe in the stationary mode results from a time variation of the field components, and is given by

$$
\begin{aligned}
V_{i}(t) & =\sum_{n=1}^{\infty} \frac{2 N_{i} L_{i} R_{r e f}}{n}\left(\frac{R_{i}}{R_{r e f f}}\right)^{n} \sin \left(\frac{n \Delta_{i}}{2}\right) \\
& \times\left[\dot{B}_{n}(t) \sin \left(n \theta_{i}\right)+\dot{A}_{n}(t) \cos \left(n \theta_{i}\right)\right]
\end{aligned}
$$

where the dot over $B_{n}$ and $A_{n}$ denotes time derivative. In a conventional rotating tangential coil, a complete angular profile is obtained by rotating the same coil. All the geometric parameters in this case are angle independent, and the cxpression for the flux, or the voltage, represents a Fourier scrics. In the case of an array of tangential coils in the stationary mode, the geometric parameters are not necessarily the same for all the coils. Consequently, the various terms in the summation in (1) or (2) are not the same as the Fouricr components of the voltage profile. Thus, a simple FFT analysis of the voltage profile assembled from the signals of all the probes does not directly give the harmonic terms.

If the geometric parameters of all the probes are nearly the same, and if the angular positions of the probes are uniformly spaced, one can apply a simple FFT analysis. Thus, it is very important to precisely match the characteristics of all the probes, not only in the fabrication of the printed circuit boards, but also in their mounting on the coil form. If this condition cannot be met, one must obtain the time derivatives of various harmonics using a fitting procedure. We plan to carry out the analysis using both approaches to check the adequacy of the FFT technique for a given coil.

The harmonic components at any time are obtained by integrating the time derivatives:

$$
B_{n}(t)=B_{n}(0)+\int_{0}^{t} \dot{B}_{n}(t) d t ; \quad A_{n}(t)=A_{n}(0)+\int_{0}^{t} \dot{A}_{n}(t) d t
$$

The initial values of the harmonics, $B_{n}(0)$ and $A_{\mathrm{n}}(0)$, can be determined, for example, using the same coil in a rotating mode before the magnet is ramped. The numerical integration can be avoided by using digital integrators instead of voltmeters to record the probe signals.

\section{ACKNOWLEDGMENT}

We are gratefill for the contributions of our design and technician staff, B. Azzara, J. Cintorino, J. McNeil, D. Oldham, A. Sauerwald, and D. Sullivan.

\section{REFERENCES}

[1] Scc, for cxamplc, A. Jain, "Harmonic coils," in Proc.CERN Accelerator School on Measurement and Alignment of Accelerator and Detector Magnets, Anacapri, Italy, April 11-17, 1997, CERN-98-05, pp.175-217.

[2] P. Spiller, et al., "The GSI synchrotron facility proposal for accelcration of high intensity ion and proton beams," Proc. 2003 Parficle Accelerator Conf., Portland, OR. USA. May 12-16. 2003. Availablc: http://warrior.lbl.gov:7778/pacfiles/papers/THURSDAY/PM ORAL/R OPA006/ROPA006.PDF

[3] P. Wanderer, et al., "Initial test of a fast-ramped superconducting model dipolc for GSI'S proposed SIS200 accelcrator," Proc. 2003 Particle Accelerator Conf., Portland, OR, USA. May 12-16, 2003. Available: http://warrior.lbl.gov:7778/pacfilcs/papcrs/WEDNESDAY/AM POSTE R/WPAB054/WPAB054.PDF

[4] N. Tsoupas, et al. "Injection accelcration and extraction of high intensity proton beam for the "Ncutrino Facility" project at BNL," Proc. 2003 Particle Accelerator Conf., Portland, OR, USA, May 12-16, 2003. Availablc:

http://warrior.Ibl.gov:7778/pacfilcs/papcrs/TUESDAY/PM_POSTER/T PPB006/TPPB006.PDF

[5] T. Ernst, et al.. "Imaging the awake animal brain," project underway at Brookhaven National Laboratory, Upton, NY, USA.

[6] W.G. Clark, J.M. Moore, and W.H. Wong, "Multiple coil pulsed magnctic rcsonance mcthod for mcasuring cold SSC dipole magnet ficld quality," Supercollider 2, Ncw York: Plcnum, 1990, pp.405-14.

[7] H. Arima, S. Kato, K. Machata, K. Ishibashi, "Multipolc analysis for absolute magnetic ficld measured by multi-probe pulsed-NMR method," JEEE Trans. Appl. Supcrconductivity, Vol. 10(1), pp.1450-3 (2000).

[8] D. Barlow, "Rotating coil measurement system for the APT/LEDA C.DTL quadrupolc magnets." IEEE Trans. Applicd Superconductivity, Vol. 10(1), pp.1447-9 (2000). 